\title{
Sero-prevalence of Hepatitis B Surface Antigen and Associated Factors Among Female Sex Workers in Hawassa, Ethiopia, 2019
}

Deresse Daka ( $\nabla$ drsdk200@gmail.com )

Hawassa University https://orcid.org/0000-0003-4146-1025

Getahun Hailemeskel

Hawassa University College of Medicine and Health Sciences

\section{Research}

Keywords: Hepatitis B virus, Female sex workers, Hawassa

Posted Date: December 7th, 2020

DOI: https://doi.org/10.21203/rs.3.rs-120559/v1

License: (c) (i) This work is licensed under a Creative Commons Attribution 4.0 International License. Read Full License 


\section{Abstract}

\section{Background}

Hepatitis B virus infection is one of the most serious infections and is a major public health problem. Female Sex Workers (FSWs) are considered to be at soaring risk for transmission and acquisition of HBV due to exposure to multiple sexual partners and barriers to the negotiation of consistent condom use. HBV screening services are not widely available in Ethiopia particularly for high risk groups such as persons with multiple sex workers. Hence, this study aimed to assess Sero-prevalence of HBVsAg and associated factors among FSWs in southern Ethiopia.

\section{Method}

A cross-sectional study was conducted from November to February 2019 at Hawassa city in ISHDO confidential clinic on 383 FSWs. A consecutive sampling technique was used to select study participants using standardized questionnaire. Data were entered to SPSS version 20.0 and analyzed for different variables using Logistic regression. HBsAg was detected from plasma samples using ELISA method.

\section{Results}

The prevalence of HBV among FSWs was 9.2\%(95\% Cl:6.3-12.1). The condom use, condom breakage during sex, use of stimulants, duration of prostitute, history of STI, history of genital ulcer, the number of clients used per day, sex during menses, sexual assault, common use of sharp material and the history of abortion were one of the common factors associated with HBV among FSWs.

\section{Conclusion}

The prevalence of HBV among FSWs in this study area was high. Condom use, history of genital ulcer, sexes during menses and sharp material sharing is highly associated with HBV infection among FSWs and emphasis should be given.

\section{Plain English Summary}

Hepatitis $B$ virus infection is one of the most serious infections and is a major public health problem. Africa has the second largest number of chronic HBV carriers after Asia and is considered as a region of high endemicity. HBV prevalence is highest in the adult population of the Western Pacific Region and Africa. Female Sex Workers (FSWs) are considered to be at soaring risk for transmission and acquisition of HBV due to exposure to multiple sexual partners and barriers to the negotiation of consistent condom use.

Of the 381 study participants who tested for Hepatitis B virus (HBV), 35(9.2\%) were positive for hepatitis B surface antigen ( $\mathrm{HBsAg}$ ) test which is more prevalent than other study population. Condom use, history of genital ulcer, sexes during menses and sharp material sharing is highly associated with HBV infection among FSWs. Therefore, efforts by both governmental and non-governmental organizations should be made to give emphasis on diagnosis and treatment of STIs, educate the community on risky sexual behaviors. Further survey should be done on larger sample size and with different study design.

\section{Background}


Hepatitis B virus (HBV) is a DNA virus belonging to the Hepadnaviridae family. It is a virus that infects the liver. ${ }^{1,2}$ HBV was discovered in 1965 when Blumberg and co-workers found the Hepatitis surface antigen which was originally called the Australia. ${ }^{3}$

As the World Health Organization (WHO) global health impact report of viral hepatitis more than 240 and 150 million populations were affected by chronic liver disease due to HBV and HCV respectively ${ }^{2}$. Africa has the second largest number of chronic HBV carriers after Asia and is considered as a region of high endemicity. ${ }^{1}$

HBV prevalence is highest in the adult population of the Western Pacific Region and Africa, where 6.2\% and 6.1\% respectively. In the Eastern Mediterranean Region, South-East Asia, Europe and population of the Americas, an estimated $3.3 \%, 2.0 \%, 1.6 \% \%$ and $0.7 \%$ of the general population are infected, respectively. ${ }^{4}$

It is mentioned that Africa is one of the continents with the highest prevalence of HBV and Ethiopia being a member of this continent shares this burden. ${ }^{2}$

HBV is most commonly spread through exposure to infected blood and various body fluids, as well as through saliva, menstrual, vaginal, and seminal fluids and reuse of needles and syringes either in healthcare settings or among persons who inject drugs. Besides, the infection can occur during medical, surgical and dental procedures, through tattooing, or through the use of razors and similar objects that are contaminated with infected blood. ${ }^{4}$

The prevalence of HBV among Female sex workers was $28.9 \%$ in Gondar, Ethiopia, ${ }^{5} 6 \%$ in Makelle, Ethiopia, ${ }^{6} 4.20 \%$ in Republic of Congo, ${ }^{7} 17.1 \%$ in Nigeria, ${ }^{8} 23.6 \%$ in São Paulo, Brazil, ${ }^{9} 10.7 \%$ in China. ${ }^{10}$

The risk factors associated with HBV among female sex workers were, workplace of sex, inconsistent condom use, and use of the drug. ${ }^{6}$ The limited economic options, having many dependents, marital disruption, low education status and hazardous alcohol use and co-infection with other sexual transmitted infections ${ }^{9}$ were common factors associated with HBV infection. Also living in the city, ${ }^{9}$ drug abuse and anal sex, ${ }^{11}$ uses of cracked condom were risk factors. ${ }^{9}$ Moreover, a history of multiple sex partners and women who have experienced child sexual abuse ${ }^{12,13}$ constitutes the potential risk factors. ${ }^{14}$ Therefore, this study aimed to determine the seroprevalence and associated factors of HBV among adolescent and young adult Female sex workers in Hawassa, integrated services on Health development (ISHDO) project confidential clinic.

\section{Methods}

\section{Study design and population}

The study was conducted at integrated services on Health development (ISHDO) project confidential clinic in Hawassa town in southern Ethiopia. It is one of the nongovernmental institutions founded to support marginalized populations to give ISHDO. Hawassa is the capital city of the South region, located on the shores of Lake Hawassa in the Great Rift Valley, $275 \mathrm{~km}$ South of Addis Ababa. Hawassa is a good tourist destination and recreational area of the south region. The town is locally called "Fikir Ketema" which means a lovely town. The study was conducted from July 1-November 31st 2019.

A cross-sectional study was conducted on adolescent and young adult Female sex workers. All adolescent and young adult female sex workers in the Hawassa town were the source of the population. About 383 female sex workers were included from eight sub-cities. All Kebeles female sex workers were pre-registered in the registration 
book. They are usually picked by special assigned ex-sex workers, namely: "Demand creator" by moving home to home and brought them to their special clinic. In this study, all Female sex workers who are living in the town and who had been working as a commercial sex worker for at least 3 months have participated. The age greater than 16 years old female sex workers were included. However, female sex workers who were living outside the town and who live less than 3 months in the city were not included in this study

\section{Specimen collection}

Three up to five milliliters $(3-5 \mathrm{ml})$ of whole blood was drawn to obtain plasma and stored at $-20^{0} \mathrm{c}$ in the upper compartment of the refrigerator until it tested. HBsAg was detected from plasma samples by using AiD $^{\mathrm{TM}}$ antibody sandwich HBsAg ELISA method (WANTAI HBV diagnostics AiD ${ }^{\text {TM }}$ HBsAg ELISA). The test was conducted following manufacturer's instructions and the Microplates read at a wavelength of $450 \mathrm{~nm}$ using the Enzyme linked immune assay (ELISA) reader. The presence or absence of HBsAg was determined by relating the absorbance of the unknown sample to the cut-off value.

\section{Statistical analysis}

Data were entered and cleaned using Epi-Data version 3.1 and exported into SPSS version 21. Binary logistic regression analysis was being performed accordingly. A p-value of less than 0.05 considered as statistical significance. The pretest was performed on $5 \%$ of the total sample size, to assure the consistency and quality of data.

\section{Result}

\section{Socio-demographic characteristics}

Out of a total of 383 enrolled subjects, 381(99.5\%) were completed information from the questionnaire and also recruited for laboratory blood tests. About $45.7 \%$ of the participants were $20-24$ ages. The mean (Mean \pm SD) age was $22.63 \pm 4.3$ and the median age was 22 . The age range was between 16 and 40 . More than three fourth of the group is single and very few $2.9 \%$ were married. The majority of $(63.5 \%)$ of the respondents were from urban. About $306(80.3 \%)$ of the study participants were attended formal education, whereas $53.5 \%$ of the participants were 5 th -8th grade. More than $35 \%$ of the study participants have the monthly income range of 1501 to 3000 Ethiopian Birr with a mean and mode of 1992.70 and 1000.00 ETB respectively (Table 1). 
Table 1

socio-demographic characteristics of study participants $2019(n=381)$

\begin{tabular}{|c|c|c|c|}
\hline Variables & & Frequency & Percent \\
\hline \multirow[t]{5}{*}{ Age in years } & $16-19$ & 91 & 23.9 \\
\hline & $20-24$ & 174 & 45.7 \\
\hline & $25-29$ & 90 & 23.6 \\
\hline & $30-34$ & 17 & 4.5 \\
\hline & $35-40$ & 9 & 2.4 \\
\hline \multirow[t]{4}{*}{ Marital status } & Married & 11 & 2.9 \\
\hline & Single & 289 & 75.9 \\
\hline & Widowed & 37 & 9.7 \\
\hline & Divorced & 44 & 11.5 \\
\hline \multirow[t]{5}{*}{ Educational Status } & No formal education & 75 & 19.7 \\
\hline & $1-4$ & 47 & 12.3 \\
\hline & $5-8$ & 204 & 53.5 \\
\hline & $9-12$ & 52 & 13.6 \\
\hline & Above 12th & 3 & .8 \\
\hline \multirow[t]{2}{*}{ Residence } & Rural & 139 & 36.5 \\
\hline & Urban & 242 & 63.5 \\
\hline \multirow[t]{4}{*}{ Religion } & Protestant & 201 & 52.8 \\
\hline & Orthodox & 164 & 43.0 \\
\hline & Muslim & 7 & 1.8 \\
\hline & Catholic & 9 & 2.4 \\
\hline \multirow[t]{3}{*}{ Living status } & Depend on family & 295 & 77.4 \\
\hline & Had another job & 75 & 19.7 \\
\hline & Other specify & 11 & 2.9 \\
\hline \multirow[t]{2}{*}{ Dependent people } & Yes & 187 & 49.1 \\
\hline & No & 194 & 50.9 \\
\hline \multirow[t]{3}{*}{ Dependent Size } & No dependents & 194 & 50.9 \\
\hline & $1-2$ & 141 & 37.0 \\
\hline & $2-4$ & 46 & 12.1 \\
\hline Average Monthly Income in ETB* & $501-1000$ & 104 & 27.3 \\
\hline
\end{tabular}




\begin{tabular}{|llll|}
\hline Variables & & Frequency & Percent \\
\hline & $1001-1500$ & 87 & 22.8 \\
\cline { 2 - 4 } & $1501-3000$ & 136 & 35.7 \\
\hline ETB; Ethiopian Birr & $3001-7000$ & 54 & 14.2 \\
\hline
\end{tabular}

About $45.1 \%$ of the sex workers were doing their sex at the hotel. Despite the condom utilization rates of the study participant were $96.3 \%$, the breakage rate of the condom was $27.1 \%$. However, $64 \%$ of them are washed with water and have no further action. Few of them are not using a condom at all (3.7\%) to get extra money. The alcohol consumption and injecting drugs/stimulants before the sex rate was $83.2 \%$ and $63.0 \%$ respectively (Table 2 ). 
Table 2

sexual behavior of the study participants $2019(n=381)$

\begin{tabular}{|c|c|c|c|}
\hline Variables & & Frequency & Percent \\
\hline \multirow[t]{4}{*}{ Place of sex } & Hotel based & 172 & 45.1 \\
\hline & Street based & 160 & 42.0 \\
\hline & Home based & 48 & 12.6 \\
\hline & Any type & 1 & .3 \\
\hline \multirow[t]{2}{*}{ Condom utilization during sex } & Yes & 240 & 63.0 \\
\hline & No & 141 & 37.0 \\
\hline \multirow[t]{3}{*}{ Frequency of condom utilization } & Always & 185 & 48.6 \\
\hline & Sometimes & 43 & 11.3 \\
\hline & Rarely & 12 & 3.1 \\
\hline \multirow[t]{3}{*}{ Reason for not using condom } & To satisfy customers & 57 & 15.0 \\
\hline & To get more money & 74 & 19.4 \\
\hline & Negligence & 10 & 2.6 \\
\hline \multirow[t]{2}{*}{ Breakage of condom during sex } & Yes & 67 & 17.6 \\
\hline & No & 173 & 45.4 \\
\hline \multirow[t]{3}{*}{ Action of breakage of condom during sex } & Went to health facility & 10 & 2.6 \\
\hline & Nothing & 23 & 6.0 \\
\hline & Washing with water & 34 & 8.9 \\
\hline \multirow[t]{2}{*}{ Alcohol consumption } & Yes & 308 & 80.8 \\
\hline & No & 73 & 19.2 \\
\hline \multirow[t]{3}{*}{ Frequency of alcohol consumption } & Always & 74 & 19.4 \\
\hline & Sometimes having sex & 156 & 40.9 \\
\hline & Rarely & 78 & 20.5 \\
\hline \multirow[t]{2}{*}{ Utilization of injectable drugs/ stimulants before sex } & Yes & 243 & 63.8 \\
\hline & No & 138 & 36.2 \\
\hline \multirow[t]{4}{*}{ Type of stimulants/injectable drugs } & Khat only & 162 & 42.5 \\
\hline & Khat and cigarette & 15 & 3.9 \\
\hline & Khat and Shisha & 40 & 10.5 \\
\hline & Shisha only & 26 & 6.8 \\
\hline Duration of prostitute commitment & $<1$ years & 65 & 17.1 \\
\hline
\end{tabular}

STI; Sexual transmitted infection; HBV; Hepatitis B virus 


\begin{tabular}{|c|c|c|c|}
\hline \multicolumn{2}{|l|}{ Variables } & \multirow{2}{*}{$\begin{array}{l}\text { Frequency } \\
249\end{array}$} & \multirow{2}{*}{$\begin{array}{l}\text { Percent } \\
65.4\end{array}$} \\
\hline & $2-5$ years & & \\
\hline & $>6$ years & 67 & 17.6 \\
\hline \multirow[t]{3}{*}{ Sexual direction/position } & vaginal sex only & 287 & 75.3 \\
\hline & vaginal and anal & 39 & 10.2 \\
\hline & vaginal and oral & 55 & 14.4 \\
\hline \multirow[t]{2}{*}{ HBV vaccination } & Yes & 1 & .3 \\
\hline & No & 380 & 99.7 \\
\hline \multirow[t]{2}{*}{ History of STI infection } & Yes & 100 & 26.2 \\
\hline & No & 281 & 73.8 \\
\hline \multirow[t]{2}{*}{ Type of STI infection } & Syphilis & 18 & 4.7 \\
\hline & Gonorrhea & 82 & 21.5 \\
\hline \multirow[t]{2}{*}{ History of Genital ulcer } & Yes & 105 & 27.6 \\
\hline & No & 276 & 72.4 \\
\hline \multirow[t]{3}{*}{ Action on Genital ulcer } & Went to a health facility & 77 & 20.2 \\
\hline & treat with herbal treatment & 23 & 6.0 \\
\hline & nothing did & 5 & 1.3 \\
\hline \multirow[t]{2}{*}{ Presence of steady partner } & Yes & 103 & 27.0 \\
\hline & No & 278 & 73.0 \\
\hline \multirow[t]{2}{*}{ Use of condom with steady partner } & Yes & 55 & 14.4 \\
\hline & No & 48 & 12.6 \\
\hline \multirow[t]{2}{*}{ Number of clients accommodated per day } & $<5$ & 224 & 58.8 \\
\hline & $\geq 5$ & 157 & 41.2 \\
\hline \multirow[t]{2}{*}{ Sex during menses } & Yes & 11 & 2.9 \\
\hline & No & 370 & 97.1 \\
\hline \multirow[t]{2}{*}{ A habit of sexual abuse/ harassment } & Yes & 44 & 11.5 \\
\hline & No & 337 & 88.5 \\
\hline \multirow[t]{2}{*}{ Have you ever received Blood transfusion? } & Yes & 10 & 2.6 \\
\hline & No & 371 & 97.4 \\
\hline \multirow[t]{2}{*}{ Sharp material for common use (tattooing) } & Yes & 30 & 7.9 \\
\hline & No & 351 & 92.1 \\
\hline
\end{tabular}

STI; Sexual transmitted infection; HBV; Hepatitis B virus 


\begin{tabular}{|llll|}
\hline Variables & & Frequency & Percent \\
\hline History of Abortion & Yes & 36 & 9.4 \\
\cline { 2 - 4 } & No & 345 & 90.6 \\
\hline Place of abortion & Health facility & 11 & 30.6 \\
\cline { 2 - 4 } & Traditionally & 25 & 69.4 \\
\hline HBV status & Positive & 35 & 9.2 \\
\cline { 2 - 4 } & Negative & 346 & 90.8 \\
\hline STl; Sexual transmitted infection; HBV; Hepatitis B virus & & \\
\hline
\end{tabular}

In this study, $65.4 \%$ of the Female sex workers involved for 2-5 years usually using vaginal sex only. However, a few of them (10.2\%) used vaginal and anal sex. Almost none of them have not vaccinated with HBV vaccination.

The history of STI among Female sex workers was $24.7 \%$ and have more than $81 \%$ of them usually infected with gonorrhea. Few of them have sex during menses (2.9\%). In this study, the habit of sexual abuse/harassment, common sharp material utilization (tattooing) and history of abortion were $11.5 \%, 7.9 \%$, and $9.4 \%$ respectively.

\section{Prevalence and determinants of HBV}

Of the 381 study participants who tested for HBV, 35(9.2\%) were positive for HBsAg test. 
Table 3

Factors associated with HBV among females sex workers at Hawassa City, Southern Ethiopia, $2019(\mathrm{n}=381)$

\begin{tabular}{|c|c|c|c|c|c|c|c|}
\hline \multicolumn{2}{|l|}{ Variables } & $\begin{array}{l}\text { HBV (+) } \\
N(\%)\end{array}$ & $\begin{array}{l}\text { HBV(-) } \\
\text { N (\%) }\end{array}$ & $\begin{array}{l}\text { COR } \\
(95 \% \mathrm{Cl})\end{array}$ & $\begin{array}{l}p- \\
\text { value }\end{array}$ & $\begin{array}{l}\text { AOR } \\
(95 \% \mathrm{Cl})\end{array}$ & $p$-value \\
\hline \multirow[t]{5}{*}{ Age in years } & $16-19$ & $5(14.3)$ & $86(24.6)$ & $\begin{array}{l}2.15(0.22 \\
20.73)\end{array}$ & \multirow[t]{5}{*}{0.06} & $0.47(0.05,4.49)$ & \multirow[t]{5}{*}{0.33} \\
\hline & $20-24$ & $16(45.7)$ & $158(45.7)$ & $\begin{array}{l}1.23(0.15 \\
10.51)\end{array}$ & & $0.81(0.09,6.90)$ & \\
\hline & $25-29$ & $10(28.6)$ & $80(23.6)$ & $1.0(0.11,8.85)$ & & $1.0(0.11,8.85)$ & \\
\hline & $30-34$ & $3(8.6)$ & $14(4.0)$ & $0.58(0.05,6.59)$ & & $\begin{array}{l}\text { 1.71(0.15, } \\
19.34)\end{array}$ & \\
\hline & $35-40$ & $1(2.9)$ & $8(2.3)$ & 1 & & 1 & \\
\hline \multirow{4}{*}{$\begin{array}{l}\text { Marital } \\
\text { status }\end{array}$} & Married & $1(2.90)$ & $10(2.9)$ & 1 & \multirow[t]{4}{*}{0.21} & 1 & \multirow[t]{4}{*}{0.07} \\
\hline & Single & $26(74.3)$ & $263(76.0)$ & $1.01(0.13,8.22)$ & & $1.00(0.10,9.96)$ & \\
\hline & Widowed & $4(11.4)$ & $33(9.5)$ & $0.83(0.82,8.25)$ & & $1.01(0.34,3.05)$ & \\
\hline & Divorced & $4(11.4)$ & $40(11.6)$ & $1.0(0.10,9.96)$ & & $0.83(0.19,3.55)$ & \\
\hline \multirow[t]{4}{*}{$\begin{array}{l}\text { Educational } \\
\text { Status }\end{array}$} & $\begin{array}{l}\text { No formal } \\
\text { education }\end{array}$ & $7(20.0)$ & 68(19.7) & $1.19(0.38,3.76)$ & \multirow[t]{4}{*}{0.09} & $0.84(0.27,2.66)$ & \multirow[t]{4}{*}{0.103} \\
\hline & $1-4$ & $3(8.6)$ & $44(12.7)$ & $1.79(0.42,7.61)$ & & $0.56(0.13,2.36)$ & \\
\hline & $5-8$ & 19(54.3) & $185(53.5)$ & $1.19(0.45,3.15)$ & & $0.84(0.32,2.21)$ & \\
\hline & $\begin{array}{l}9 \text { and } \\
\text { above }\end{array}$ & $6(17.1)$ & $49(14.2)$ & 1 & & 1 & \\
\hline \multirow[t]{2}{*}{ Residence } & Rural & $5(14.3)$ & $124(35.8)$ & 1 & \multirow[t]{2}{*}{0.015} & 1 & \multirow[t]{2}{*}{0.022} \\
\hline & Urban & $30(85.7)$ & $222(64.2)$ & $0.29(0.11,0.79)$ & & $0.74(0.37,1.51)$ & \\
\hline \multirow{2}{*}{$\begin{array}{l}\text { Living status } \\
\text { depend on }\end{array}$} & Family & $31(88.6)$ & 188(54.3) & 1 & \multirow[t]{2}{*}{0.001} & $0.98(0.43,2.25)$ & \multirow[t]{2}{*}{0.14} \\
\hline & $\begin{array}{l}\text { Not on } \\
\text { family }\end{array}$ & $4(11.4)$ & $158(54.7)$ & $6.51(2.25,18.85)$ & & 1 & \\
\hline \multirow{2}{*}{$\begin{array}{l}\text { Having } \\
\text { dependent } \\
\text { people }\end{array}$} & Yes & $28(80.0)$ & $159(46.0)$ & 1 & \multirow[t]{2}{*}{0.00} & 1 & \multirow[t]{2}{*}{0.12} \\
\hline & No & $7(20.0)$ & $187(54.0)$ & $\begin{array}{l}4.70(2.00 \\
11.06)\end{array}$ & & $1.28(0.43,3.84) \star$ & \\
\hline \multirow{4}{*}{$\begin{array}{l}\text { Monthly } \\
\text { Income }\end{array}$} & $501-1000$ & $8(22.9)$ & $90(26.0)$ & $0.99(0.20,2.39)$ & \multirow[t]{4}{*}{0.23} & & \multirow[t]{4}{*}{0.25} \\
\hline & $\begin{array}{l}1001- \\
1500\end{array}$ & $15(42.9)$ & $62(17.9)$ & $0.69(0.08,0.81)$ & & & \\
\hline & $\begin{array}{l}1501- \\
3000\end{array}$ & $8(22.9)$ & 129(37.3) & $0.25(0.29,3.42)$ & & & \\
\hline & $\begin{array}{l}3001- \\
7000\end{array}$ & $4(11.4)$ & $65(18.8)$ & 1 & & & \\
\hline
\end{tabular}




\begin{tabular}{|c|c|c|c|c|c|c|c|}
\hline \multirow[t]{2}{*}{ Variables } & & HBV (+) & HBV(-) & COR & \multirow{2}{*}{$\begin{array}{l}p- \\
\text { value }\end{array}$} & AOR & \multirow[t]{2}{*}{$p$-value } \\
\hline & & $\mathbf{N}(\%)$ & $\mathrm{N}(\%)$ & $(95 \% \mathrm{Cl})$ & & $(95 \% \mathrm{Cl})$ & \\
\hline \multirow[t]{3}{*}{$\begin{array}{l}\text { Dependent } \\
\text { Size }\end{array}$} & $\begin{array}{l}\text { No } \\
\text { dependents }\end{array}$ & $7(20.0)$ & 187(54.0) & 1 & \multirow[t]{3}{*}{0.00} & $0.94(0.34,2.61)$ & \multirow[t]{3}{*}{0.08} \\
\hline & $1-2$ & $23(65.7)$ & 118(34.1) & $0.19(0.80,0.46)$ & & $0.55(0.17,1.84)$ & \\
\hline & $2-4$ & $5(14.3)$ & $41(11.8)$ & $0.31(0.09,1.02)$ & & 1 & \\
\hline \multirow[t]{2}{*}{ Condom use } & Yes & 10(28.6) & $230(66.5)$ & 1 & \multirow[t]{2}{*}{0.00} & 1 & \multirow[t]{2}{*}{$0.01^{*}$} \\
\hline & No & $25(71.4)$ & 116(33.5) & $\begin{array}{l}4.96(2.30 \\
10.67)\end{array}$ & & $\begin{array}{l}\text { 6.38(2.04, } \\
18.51)\end{array}$ & \\
\hline \multirow{2}{*}{$\begin{array}{l}\text { Condom } \\
\text { Breakage } \\
\text { during sex } \\
(n=240)\end{array}$} & Yes & $6(60.0)$ & $61(26.5)$ & $\begin{array}{l}\text { 4.16(1.13, } \\
15.23)\end{array}$ & \multirow[t]{2}{*}{0.03} & $2.10(0.95,4.62)$ & \multirow[t]{2}{*}{$0.03^{*}$} \\
\hline & No & $4(40.0)$ & 169(73.5) & 1 & & 1 & \\
\hline \multirow{2}{*}{$\begin{array}{l}\text { Alcohol } \\
\text { consumption }\end{array}$} & Yes & $21(60.0)$ & $287(82.9)$ & $3.24(1.56,6.74)$ & \multirow[t]{2}{*}{0.00} & $1.21(0.45,3.26)$ & \multirow[t]{2}{*}{0.14} \\
\hline & No & 14(40.0) & $59(17.1)$ & 1 & & 1 & \\
\hline \multirow[t]{2}{*}{$\begin{array}{l}\text { Use of } \\
\text { stimulant }\end{array}$} & Yes & $29(82.9)$ & $214(61.8)$ & $2.98(1.21,7.37)$ & \multirow[t]{2}{*}{0.013} & $\begin{array}{l}3.25(1.59 \\
18.63)\end{array}$ & \multirow[t]{2}{*}{$0.018 *$} \\
\hline & No & $6(17.1)$ & 132(38.2) & 1 & & 1 & \\
\hline \multirow{3}{*}{$\begin{array}{l}\text { Duration of } \\
\text { prostitution } \\
\text { service }\end{array}$} & $<1$ & $3(8.6)$ & 62(17.9) & $\begin{array}{l}4.74(1.30 \\
17.34)\end{array}$ & \multirow[t]{3}{*}{0.00} & 1 & \multirow[t]{3}{*}{$0.02^{*}$} \\
\hline & $2-5$ & $18(51.4)$ & $223(64.5)$ & $2.84(1.34,6.04)$ & & $2.03(0.49,8.50)$ & \\
\hline & $>6$ & 14(40.0) & 61(17.6) & 1 & & $0.84(0.33,2.14)$ & \\
\hline \multirow[t]{3}{*}{$\begin{array}{l}\text { Type of sex } \\
\text { usually used }\end{array}$} & $\begin{array}{l}\text { Vaginal } \\
\text { only }\end{array}$ & $\begin{array}{l}24( \\
68.6)\end{array}$ & $263(76.0)$ & 1 & \multirow[t]{3}{*}{0.08} & $1.20(0.34,4.26)$ & \multirow[t]{3}{*}{0.98} \\
\hline & $\begin{array}{l}\text { Vaginal } \\
\text { and anal }\end{array}$ & $5(14.3)$ & $34(9.8)$ & $0.62(0.22,1.73)$ & & $0.75(0.29,1.92)$ & \\
\hline & $\begin{array}{l}\text { Vaginal } \\
\text { and oral }\end{array}$ & $6(17.1)$ & $49(14.2)$ & $0.75(0.29,1.92)$ & & 1 & \\
\hline \multirow[t]{2}{*}{ Vaccinated } & Yes & $1(2.9)$ & $1(0.3)$ & 1 & \multirow[t]{2}{*}{0.18} & 1 & \multirow[t]{2}{*}{0.23} \\
\hline & No & $34(97.1)$ & $345(99.7)$ & $\begin{array}{l}\text { 10.15(0.62 } \\
165.88)\end{array}$ & & $0.99(0.06,1.61)$ & \\
\hline \multirow[t]{2}{*}{$\begin{array}{l}\text { History of } \\
\text { STI }\end{array}$} & Yes & $20(57.1)$ & $80(23.1)$ & $4.43(2.17,9.06)$ & \multirow[t]{2}{*}{0.00} & $\begin{array}{l}2.15(1.02 \\
6.93)^{\star}\end{array}$ & \multirow[t]{2}{*}{$0.011^{*}$} \\
\hline & No & $15(42.9)$ & $266(76.9)$ & 1 & & 1 & \\
\hline \multirow[t]{2}{*}{$\begin{array}{l}\text { History of } \\
\text { Genital ulcer }\end{array}$} & Yes & 18(51.4) & $87(25.1)$ & $3.15(1.56,6.39)$ & \multirow[t]{2}{*}{0.00} & $\begin{array}{l}\text { 4.64(1.31, } \\
11.35)\end{array}$ & \multirow[t]{2}{*}{$0.004^{* *}$} \\
\hline & No & 17(48.6) & $259(74.9)$ & 1 & & 1 & \\
\hline
\end{tabular}

** Significant at $\mathrm{p}<0.005,{ }^{*}$ significant at $\mathrm{p}<0.05 ; \mathrm{AOR}$, adjusted odds ratio; $\mathrm{COR}$, crude odds ratio; $\mathrm{Cl}$, confidence interval; OR, odds ratio 


\begin{tabular}{|c|c|c|c|c|c|c|c|}
\hline \multicolumn{2}{|l|}{ Variables } & $\begin{array}{l}\mathrm{HBV}(+) \\
\mathrm{N}(\%)\end{array}$ & $\begin{array}{l}\text { HBV(-) } \\
\mathrm{N}(\%)\end{array}$ & $\begin{array}{l}\text { COR } \\
(95 \% \mathrm{Cl})\end{array}$ & $\begin{array}{l}p- \\
\text { value }\end{array}$ & AOR & $p$-value \\
\hline \multirow{3}{*}{$\begin{array}{l}\text { Number of } \\
\text { clients used } \\
\text { per day }\end{array}$} & & & & & \multirow{3}{*}{0.00} & & \multirow{3}{*}{$0.018 *$} \\
\hline & $<5$ & $9(25.7)$ & $202(58.4)$ & 1 & & 1 & \\
\hline & $\geq 5$ & $26(74.3)$ & $144(41.6)$ & $4.05(1.84,8.91)$ & & $3.25(1.59,7.47)$ & \\
\hline \multirow{2}{*}{$\begin{array}{l}\text { Sexes during } \\
\text { menses }\end{array}$} & Yes & $4(11.4)$ & $7(2.0)$ & $6.25(1.7,22.53)$ & \multirow[t]{2}{*}{0.00} & $5.85(1.29,21.44)$ & \multirow[t]{2}{*}{$0.002 * *$} \\
\hline & No & $31(88.6)$ & $339(98.0)$ & 1 & & 1 & \\
\hline \multirow{2}{*}{$\begin{array}{l}\text { Sexual } \\
\text { Assault }\end{array}$} & Yes & $9(25.7)$ & $35(10.1)$ & $3.08(1.34,7.09)$ & \multirow[t]{2}{*}{0.00} & $2.93(1.23,9.01)$ & \multirow[t]{2}{*}{$0.007^{* *}$} \\
\hline & No & $26(74.3)$ & $311(89.9)$ & 1 & & 1 & \\
\hline \multirow{2}{*}{$\begin{array}{l}\text { Blood } \\
\text { received }\end{array}$} & Yes & $2(5.7)$ & $8(2.3)$ & 1 & \multirow[t]{2}{*}{0.12} & 1 & \multirow[t]{2}{*}{0.08} \\
\hline & No & $33(94.3)$ & $338(97.7)$ & $\begin{array}{l}2.56(0.52 \\
12.56)\end{array}$ & & $0.39(0.08,1.92)$ & \\
\hline \multirow[t]{2}{*}{$\begin{array}{l}\text { Use of sharp } \\
\text { material }\end{array}$} & Yes & $8(22.9)$ & $22(6.4)$ & $\begin{array}{l}\text { 4.36(1.78, } \\
10.73)\end{array}$ & \multirow[t]{2}{*}{0.00} & $4.98(1.34,10.95)$ & \multirow[t]{2}{*}{$0.008 * \star$} \\
\hline & No & 27(77.1) & $324(93.6)$ & 1 & & 1 & \\
\hline \multirow[t]{2}{*}{$\begin{array}{l}\text { History of } \\
\text { Abortion }\end{array}$} & Yes & $9(25.7)$ & $30(8.7)$ & $3.65(1.67,8.49)$ & \multirow[t]{2}{*}{0.00} & $\begin{array}{l}2.46(1.18, \\
12.19)\end{array}$ & \multirow[t]{2}{*}{$0.00 * *$} \\
\hline & No & $26(74.3)$ & $316(91.3)$ & 1 & & 1 & \\
\hline
\end{tabular}

The condom use, condom breakage during sex, use of stimulants, duration of prostitute, History of STI, history of genital ulcer, the number of clients used per day, sex during menses, sexual assault, a common use of sharp material and the history of abortion were one of the common factors associated with HBV among Female sex workers (Table 3).

In binary logistic regression analysis, common use of Condom use, ( $A O R=6.38, \mathrm{Cl} 2.04-18.51)$, Condom breakage during sex, $(A O R=2.10, \mathrm{Cl} 0.95-4.65)$, Use of stimulants, $(A O R=1.25, \mathrm{Cl} 0.59-2.63)$, History of $\mathrm{STI},(\mathrm{AOR}=2.15, \mathrm{Cl}$ 1.02-6.93), History of Genital ulcer, (AOR $=4.64, \mathrm{Cl} 1.31-11.35)$, Number of clients used per day, $(A O R=3.25, \mathrm{Cl}$ 1.59-7.47), sexes during menses ( $A O R=5.85, \mathrm{Cl}(1.29-21.44)$, sexual assault $(A O R=2.93, \mathrm{Cl} 1.23-9.01)$, sharp material sharing, $(A O R=4.98, \mathrm{Cl} 1.34-10.95)$ and History of abortion, $(A O R=2.46, \mathrm{Cl} 1.18,12.19)$, remained statistically significant factors associated with HBV infection.

\section{Discussion}

Globally the prevalence of HBV infection can be divided into high ( $>8 \%)$, intermediate $(2-8 \%)$ and low $(<2 \%)$. Hence as the prevalence of HBV among Female sex workers in this study area found to be $9.2 \%$, the prevalence is labeled as high occurrence.

This study revealed that the overall prevalence of HBV among female sex workers in hawass city was $9.2 \%$ (Cl: $95 \%$ 6.3-12.1). This is lower than the study conducted in Gondar which was $11.9 \%,{ }^{5}$ Nigeria $17.1 \%,{ }^{8}$ Cameroon $36 \%,{ }^{15}$ 
Argentina 14.4\%, ${ }^{16}$ Shanghai, China $12.3 \%,{ }^{17}$ in Goiânia, Brazil 17.1\% ${ }^{18}$ and Southern Brazil 23.1\%. ${ }^{19}$ On the other hand this study is higher than the study conducted in Mekelle, Ethiopia 6\%, ${ }^{6}$ Iran $1.2 \%,{ }^{20}$ Rwanda $2.5 \%,{ }^{21}$ Congo $7.3 \%,{ }^{7}$ Italy $3.5 \%,{ }^{22}$ Venezuela $3.8 \%,{ }^{23}$ and Afghanistan $6.54 \% .{ }^{11}$ This variation of findings across various studies might be due to diagnostic tools and reporting biases, differences in socio-demographic, differences in socio economic environments. Prevalence estimates also are likely to be influenced by stigma and discrimination and also study settings. Also unemployment status of the females might be enforced to engage in risky sexual services. ${ }^{11}$ Also, the history of previous vaccination, high prevalence of HBV among general population, use of illegal injecting drugs, consumption of alcohol, breaking of the condom during sex, a long period of prostitute engagement, anal sex and the presence of other sexual transmitted infections among female sex workers may be contributed. ${ }^{24,25}$

The major determinants of HBV were, low adherence to the condom use, history of genital ulcer, history of sexual transmitted infections, sexes during menses, sexual assault and shared use of sharp materials were statistically significant $(p<0.05)$. This is similar to the study conducted in Nigeria. ${ }^{8}$

In agreement with WHO, 2014 Prevention and Treatment of HIV and other Sexually Transmitted Infections for Sex Workers in Low and Middle-income Countries and studies result from Teresina-PI, Northeastern Brazil, India and Afghanistan ${ }^{26-29}$ this study indicated number of clients (> 5 clients (sexual partner) per week) significantly associated with HBV $[A O R=3.25,(1.59,7.47)]$. The possible explanation may be multiple clients produce greater vulnerability to risks for low adherence to the use of condoms in all sexual relations. Also, clients of sex workers may practice risky behaviors like injectable drug use etc., thus increase the risk of acquiring STIs including hepatitis $B$ virus.

Studies indicated that History of STI were predictors of exposure to HBV. ${ }^{18,20,30}$ Consistently, result of this study reflected the odds of participants who have history of STI which were more than 2 times to be positive as compared with participants who don't have $[A O R=2.15,(1.02,6.93)]$. The most common history of STI was Neisseria gonorrhea and syphilis. Also genital ulcers were seen among $27.6 \%$ participants. These infections can cause inflammation and ulcer around the genital area. Thus, it may increase the risk of becoming infected with HBV.

In this study, educational status, history of blood transfusion and alcohol consumption were not statically significant association with HBV as other studies done in Teresina-PI, Northeastern Brazil among sex workers, in three Afghan cities among female sex workers, in Mekelle among commercial sex workers and in Tehran, Iran among female sex workers found out. $6,9,20,27,28$

Even though the female sex workers who did not vaccinated previously for HBV were more in number for HBV infection than those who vaccinated, there is no statistical significant association in this study. However, similar studies were done in Argentina shown that none vaccinated people developed HBV infection more likely than vaccinated peoples. ${ }^{16}$ This indicates that vaccination is one of the prevention mechanisms of HBV infection.

According to this study, those who have previous sexual transmitted infection especially syphilis were statistically significant with the infection of HBV which is similar to the study done in, Iran and other different countries. ${ }^{18,20,30}$ Also Female sex workers who are not avoiding sex during menses were more than five times affected by HBV infection which is inconsistent with the study of Mekelle, Ethiopia. ${ }^{6}$ This study also showed that previous sexual assaulted female sex workers had more than two times the probability to develop HBV infection than those of not harassed. This correlates with studies from North America and South Africa have shown that women who have experienced sexual abuse are more likely to acquire HBV infection. ${ }^{12,13}$

Page $13 / 16$ 
As study done in Northeastern Brazil 27 sharing of sharp materials for tattooing and another purpose is significantly influencing HBV infection among female sex workers $(p<0.05)$ in this study area. Also history of genital ulcer and the habit of unsafe abortion was statistically significant among female sex workers to acquire HBV. ${ }^{18}$

Also, age, marital status, residence, monthly income, presence of steady partners, and type of sex usually used has no statistically significant association in this study area. This is similar to the study of Mekelle, Ethiopia. ${ }^{6,18}$

\section{Conclusion}

The prevalence of HBV among FSWs in this study area was high. Condom use, history of genital ulcer, sexes during menses and sharp material sharing is highly associated with HBV infection among FSWs and emphasis should be given. Since this finding indicates HBV infection is more prevalent among FSWs, efforts by both governmental and non-governmental organizations should be made to give emphasis on diagnosis and treatment of STIs, educate the community on risky sexual behaviors. Further survey should be done on larger sample size and with different study design.

\section{Abbreviations}

ISHDO: integrated services on Health development; HBV: hepatitis B virus; HBsAg: Hepatitis B surface antigen test; FSW: Female sex workers; STIs: sexual transmitted infections; AOR: Adjusted odds ration; COR: crude odds ratio; WHO: World Health Organization

\section{Declarations}

\section{Ethics approval and consent to participate}

Ethical approval was obtained from the Hawassa University Institutional Review Board Committee. A permission letter was granted from the Southern Nation, Nationality and People Regional Health Bureau to respective health institutions. Written consent was obtained from each participant prior to data collection. After informing each respondent about the aim and purpose of the study, participants were asked for their voluntary participation. Those respondents were assured they could withdraw from the study anytime if they felt unhappy. They were also informed that all data obtained from them would be kept confidential by using codes instead of any personal identifiers.

\section{Consent for publication}

Not applicable

\section{Availability of data and material}

All data and materials in this manuscript could be deposited in publicly available repositories. All relevant data is included in the paper, the full data available from the Family Guidance Association (FGA) of the Southern Ethiopia, Hawassa.

\section{Competing interests}

The authors have declared that no competing interests exist.

\section{Funding}


This study was supported by Hawassa University, college of Medicine and Health Sciences. The support included payment for data collectors and purchase of materials and supplies required for the study. The support did not include designing of the study, analysis, interpretation of data, manuscript preparation and publications.

\section{Author contributions}

DD: Conceived and designed the study, analyzed the data, involved in manuscript preparation, Involved in protocol development and manuscripts write up. GHM, Conceived and designed the study, supervised the study, involved in analysis and manuscript preparation. All authors read and approved the final manuscript.

\section{Acknowledgments}

We are grateful to the Hawassa University for technical and financial support. We would also like to thank the Integrated Service on Health and Development Organization (ISHDO) for their guidance and support during the data collection process.

\section{References}

1. WHO, World Health Organization. World Health Statistics. 2013.

2. Hwang EW, Cheung R. Global epidemiology of hepatitis B virus (HBV) infection. N Am J Med Sci 2011;4:7-13.

3. Blumberg BS, Visnich SA. A new antigen in leukemia sera JAMA 1965;191:541-546.

4. Fiona Scorgie, Matthew F. Chersich, Innocent Ntaganira, et al. Socio Demographic Characteristics and Behavioral Risk Factors of Female Sex Workers in Sub-Saharan Africa: A Systematic Review. AIDS Behav 2012;16:920-933.

5. Feleke M, Yenew K, Afework K, et al. Sero prevalence of HIV, Hepatitis B infections and Syphilis among street dwellers in Gondar city, Northwest Ethiopia. Ethiop J health Dev 2006;20.

6. Gessessew Bugussa, Berhe Dessalegn, Balem Dimitu, et al. Prevalence and factors associated with HIV and Hepatitis B virus infection among female commercial sex workers in Mekelle, Ethiopia: Cross sectional study. IJPSR 2015;6:135-146.

7. Fabien Roch Niama, Nadia Claricelle Loukabou Bongolo, Pembe Issamou Mayengue, et al. A study on HIV, Syphilis, and Hepatitis $\mathrm{B}$ and $\mathrm{C}$ virus infections among female sex workers in the Republic of Congo $B M C$, Archives of Public Health 2017;75.

8. Forbi J.C, Onyemauwa N, Gyar S.D, et al. High prevalence of Hepatitis B Virus Among female sex workers in Nigeria. Rev Inst Med trop S Paulo 2008;50:219-221.

9. Afonso Dinis Costa Passos, José Fernando de Castro Figueiredo, Ana de Lourdes Candolo Martinelli, et al. Hepatitis B among female sex workers in Ribeirão Preto - São Paulo, Brazil. REv, Bras Epidemiol 2007;10:517524.

10. Shu Su, Eric PF. Chow, Kathryn E. Muessig, et al. Sustained high prevalence of viral hepatitis and sexually transmissible infections among female sex workers in China: a systematic review and meta-analysis $B M C$ Infectious Diseases 2016;16.

11. Catherine S. Todd, Abdul Nasir, Mohammad R. Stanekzai, et al. HIV, hepatitis B, and hepatitis $C$ prevalene and associated risk behaviors among female sex workers in three Afghan Cities. NIH, AIDS2010;24:S69-S75.

12. Kalichman SC, Simbayi LC. Sexual assault history and risks for sexually transmitted infections among women in an African township in Cape Town, South Africa. AIDS care 2004;16:681-689. 
13. Wingood GM, Diclemente RJ. Child sexual abuse, HIV sexual risk and gender relations of African-American women. Amer J prev Med 1997;13:380-384.

14. Pando MA, Berini $C$, Bibini M, et al. Prevalence of HIV and other sexually transmitted infections among female commercial sex workers in Argentina Am J Trop Med Hyg 2006;74:233-238.

15. President's Emergency Plan for AIDS Relief (PEPFAR). Cameroon Operational plan report. 2011.

16. María A. Pando, Carolina Berini, Mariel Bibini, et al. Prevalence of HIV and other Sexually Transmitted Infections among female commercial sex workers in Argentina. Am J Trop Med Hyg 2006;74:233-238.

17. Zhang T, Yang Y, Yu F, et al. Kaposis sarcoma associated herpesvirus infection among female sex workers and general population women in Shanghai, China: a cross-sectional study. BMC infectious diseases 2014;14:58.

18. Matos MAd, França DDdS, Carneiro MAdS, et al. Viral hepatitis in female sex workers using the RespondentDriven Sampling. Revista de saude publica 2017;51.

19. Fabiana Schuelter-Trevisol, Geisiane Custodio, Ana Carolina Barreto da silva, et al. HIV, hepatitis B and C, and syphilis prevalence and coinfection among sex workers in Southern Brazil. Revista da Sociedade Brasileira Tropical 2013;46:493-497.

20. Moayedi-Nia S, Bayat Jozani Z, Esmaeeli Djavid G, et al. HIV, HCV, HBV, HSV, and syphilis prevalence among female sex workers in Tehran, Iran, by using respondent-driven sampling. AIDS care 2016;28:487-490.

21. Mutagoma M, Nyirazinyoye L, Sebuhoro D, et al. Syphilis and HIV prevalence and associated factors to their coinfection, hepatitis $B$ and hepatitis $C$ viruses prevalence among female sex workers in Rwanda BMC infectious diseases 2017;17:525.

22. Monica Zermiani, Carlo Mengoli, Claudia Rimondo, et al. Prevalence of Sexually Transmitted Diseases and Hepatitis C in a Survey of Female Sex Workers in the North-East of Italy The Open AIDS Journal 2012;6:60-64.

23. Camejo MI, Mata G, Diaz M. Prevalence of hepatitis B, hepatitis C and syphilis in female sex workers in Venezuela. Rev Saúde Publica 2003;37.

24. Juárez-Figueroa L, Uribe-Salas F, Conde-Glez C, et al. Low prevalence of hepatitis B markers among Mexican female sex workers. Sex Transm Inf. 741998;6:448-450.

25. Hyams KC, Escamilla J, Romero RL, et al. Hepatitis B infection in a nondrug abusing prostitute population in Mexico. Scand J Infect Dis 1990;22:527-531.

26. World Health Organization. Joint United Nations Programme on HIV/AIDS. Prevention and treatment of HIV and other sexually transmitted infections for sex workers in low-and middle-income countries: recommendations for a public health approach. Geneva, Switzerland: WHO/UNAIDS, 2014.

27. de Lima Brito Magalhães R, Moura Carvalho V, Ibiapina Brito GM, et al. Risk practices and immunization against hepatitis B among female sex workers. Revista da Rede de Enfermagem do Nordeste 2016;17.

28. Todd CS, Nasir A, Stanekzai MR, et al. HIV, hepatitis B, and hepatitis C prevalence and associated risk behaviors among female sex workers in three Afghan cities. AIDS (London, England) 2013;24

29. Desai Praseeda S, Anuradha D. A Study on the HBV and the HCV Infections in Female Sex Workers and their CoInfection with HIV. Journal of clinical and diagnostic research: JCDR 2013;7:234.

30. Adisesh M, Natesan T, Elumalai S, et al. A seroprevalence evaluation study of Hepatitis B virus, Hepatitis C virus and Herpes Simplex Virus-2 among a special study group. International Journal of Medical Research and Health Sciences 2016;5:196-199. 